\title{
Barreiras e desafios para melhoria da integração interfuncional entre Desenvolvimento de Produto e Planejamento e Controle da Produção em ambiente Engineering-to-Order
}

\section{Barriers and challenges to improve interfunctional integration between Product Development and Production Planning and Control in Engineering-to-Order Environment}

\author{
José Henrique de Andrade ${ }^{1}$ \\ Flávio Cesar Faria Fernandes ${ }^{1}$
}

\begin{abstract}
Resumo: A integração interfuncional é apontada por diversos trabalhos como uma alternativa para o aumento da excelência operacional, melhorando o desempenho organizacional por meio da melhoria da interação e colaboração nos processos organizacionais. O objetivo deste artigo é apresentar e discutir barreiras e desafios para melhoria da integração interfuncional entre Desenvolvimento de Produto (DP) e Planejamento e Controle da Produção (PCP) em Sistemas de Produção que adotam a estratégia de resposta à demanda Engineering-to-Order (ETO). Para atingir o objetivo proposto, foram utilizados um referencial teórico sobre os temas de interesse e constatações obtidas a partir do estudo de caso de três empresas metal mecânicas de grande porte que projetam e fabricam bens de capital sob encomenda. Como principais resultados, foram observadas barreiras e desafios relacionados à interação entre as áreas funcionais durante o processo de atendimento de pedido e a necessidade de ações que busquem melhorar a interação e colaboração entre DP e PCP, levando em conta as peculiaridades do ambiente ETO.
\end{abstract}

Palavras-chave: Desenvolvimento de Produto; Planejamento e Controle da Produção; Integração interfuncional; Engineering-to-Order.

\begin{abstract}
Interfunctional integration is indicated by several studies as an alternative to increased operational excellence, improving organizational performance through improved interaction and collaboration in organizational processes. The focus of this paper is to present and discuss barriers and challenges to improve interfunctional integration between Product Development (PD) and Production Planning and Control (PPC) in production systems that adopt the strategy of response to demand Engineering-to-Order (ETO). To reach that goal, we used a literature review on the topics of interest and findings obtained from the case studies of three large metal mechanical companies that design and manufacture customized-made capital goods. The following were observed as main results: barriers and challenges related to the interaction between functional areas during the process of order fulfilment, and the need for actions that seek to improve the interaction and collaboration between PD and PPC, taking into account the peculiarities of the ETO environment.
\end{abstract}

Keywords: Product Development; Production Planning and Control; Interfunctional integration; Engineering-to-Order.

\section{Introdução}

Trabalhos da área de Administração enfatizam o conjunto de mudanças significativas observadas no ambiente de negócios nas últimas décadas, ocasionadas por alterações no mercado (perfil dos consumidores, características dos produtos e serviços, condições econômicas e políticas, entre outros) e por um novo posicionamento competitivo das organizações (Sipper \& Bulfin, 1997; Corrêa \& Gianesi, 2007;
Hayes et al., 2008). Assim sendo, existe a necessidade de outro posicionamento da Administração.

Uma forma de obter esse novo posicionamento é por meio da revisão das práticas de gestão e dos processos operacionais das organizações, tornando-os coerentes com as metas estratégicas, aplicando sistemas de informação alinhados à nova realidade e procedimentos estruturados que permitam, por exemplo, maior agilidade e flexibilidade na execução

${ }^{1}$ Departamento de Engenharia de Produção, Universidade Federal de São Carlos - UFSCar, Rodovia Washington Luís, Km 235, CEP 13565-905, São Carlos, SP, Brasil, e-mail: andrade.josehenrique@gmail.com; dfcf@ufscar.br

Recebido em Nov. 7, 2013 - Aceito em Maio 5, 2015

Suporte financeiro: Este trabalho contou com suporte financeiro da CAPES e FAPESP, na forma de bolsa de estudo em nível de Doutorado. 
das atividades do negócio. Destaca-se a integração como uma forma de melhorar os processos organizacionais (Kahn, 1996; Paiva et al., 2011; Chen et al., 2013).

De acordo com Jugend (2010, p. 1), a integração pode ser definida como o " [...] trabalho colaborativo e interativo entre diferentes departamentos e/ou especialistas presentes em uma empresa que necessitam realizar trabalhos conjuntos com o propósito de atingir determinados objetivos comuns".

O Desenvolvimento de Produto (DP) trata da transformação de requisitos de mercado e de clientes em especificações de produtos, enquanto o Planejamento e Controle da Produção (PCP) trata das providências necessárias para a efetiva transformação das especificações geradas no DP em produtos que serão produzidos e entregues ao mercado consumidor, compatibilizando de forma efetiva a demanda de mercado e a capacidade de produção.

Neste sentido, promover a integração entre DP e PCP torna-se crucial, na medida em que ambos têm importância significativa nas organizações industriais, tendo em vista que realizam atividades-chave de um Sistema de Produção.

Diante deste contexto, o objetivo deste trabalho é apresentar e discutir barreiras e desafios para melhoria da integração interfuncional entre DP e PCP em Sistemas de Produção que adotam a estratégia de resposta à demanda Engineering-to-Order (ETO). Para atingir o objetivo proposto, foram utilizados um referencial teórico sobre os temas de interesse (seção 2) e dados obtidos a partir do estudo de casos de três empresas metal mecânicas de grande porte que projetam e fabricam bens de capital sob encomenda (seção 3). Por fim, são apresentados barreiras e desafios para melhoria da integração entre DP e PCP em ambiente ETO (seção 4), seguidos das conclusões (seção 5) e referências bibliográficas.

Destacam-se, como aspectos que justificam a relevância do presente trabalho, a escassa literatura sobre gestão em Sistemas de Produção em ambiente ETO e a importância do setor metal mecânico como segmento de fundamental importância para aumento da competitividade da indústria brasileira e geração de riquezas - aspecto apresentado na seção $2.3 \mathrm{com}$ base em dados da indústria de bens de capital.

\section{Referencial teórico}

\subsection{Desenvolvimento de Produto, PCP e Ambiente ETO}

Conforme já destacado, DP e PCP são funções-chave em um Sistema de Produção. Groover (2011) define um Sistema de Produção como um conjunto de pessoas, equipamentos e procedimentos organizados para realizar as operações de manufatura de uma companhia. Esse autor sugere a divisão de um Sistema de Produção em duas categorias ou níveis, a saber: 1.
Instalações - refere-se às instalações físicas do Sistema de Produção, compostas pela fábrica, equipamentos e a forma como estes equipamentos estão organizados; 2. Sistema de suporte à manufatura - refere-se ao conjunto de procedimentos usados pela companhia para administrar a produção e resolver problemas técnicos e logísticos a fim de assegurar padrões de qualidade requeridos.

De outro modo, Fernandes \& Godinho (2010, p. 1) definem-no como

[...] um conjunto de elementos (humanos, físicos e procedimentos gerenciais) inter-relacionados que são projetados para gerar produtos finais cujo valor supere o total de custos incorridos para obtê-los.

Diversos autores, ao analisarem um Sistema de Produção, buscam identificar suas atividades ou funções-chave. Slack et al. (2002), por exemplo, segmentam as atividades primárias de um Sistema de Produção em Vendas/Marketing, Produção e Desenvolvimento do Produto. De outro modo, Dilworth (1993), identifica como funções primárias, de um Sistema de Produção, Marketing, Finanças e Produção - das quais depende a obtenção de todo o potencial da organização.

Groover (2011) agrupa as funções-chave de um Sistema de Produção de manufatura naquilo que ele denomina como sistema de suporte à manufatura e o decompõe em quatro funções-chave para o negócio: 1) Funções de negócio; 2) Projeto do Produto; 3) Planejamento da manufatura; 4) Controle da manufatura. $\mathrm{O}$ autor ressalta que a interação entre estas quatro funções se dá por meio de um ciclo de processamento da informação, conforme mostra a Figura 1.

Apesar de possuírem objetivos funcionais distintos e muitas vezes até conflitantes, as funções organizacionais que compõem um Sistema de Produção, não só as primárias ou chave, devem convergir no sentido de produzir bens e serviços que atendam à necessidade do mercado consumidor e gerar valor excedente para

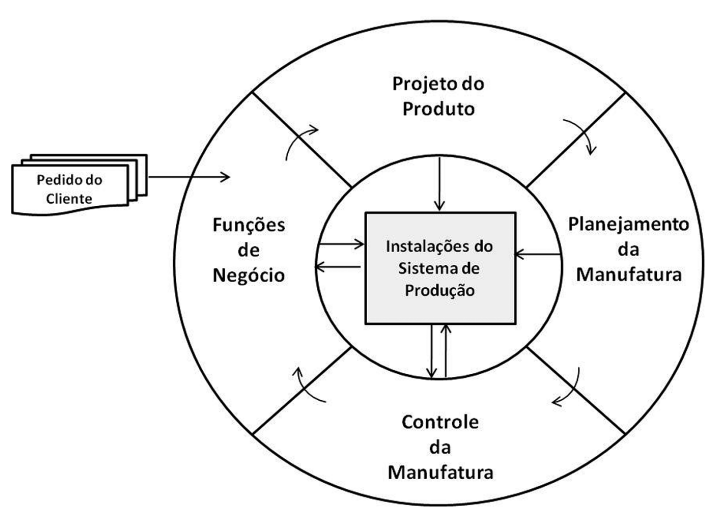

Figura 1. Ciclo de processamento da informação em um sistema típico de manufatura. Fonte: Groover (2011, p. 5). 
a manutenção da organização. Ressalta-se, ainda, que o sucesso competitivo advém da forma como as funções básicas se relacionam (Groover, 2011).

O DP responsabiliza-se pelo atendimento de requisitos técnicos, operacionais e estatutários e sua consequente transposição na forma de conhecimentos explícitos-desenhos, especificações técnicas, instruções, entre outros - os quais carregam a concepção de um produto e permitem sua construção (Amaral, 2001). Reconhece-se na literatura que de $75 \%$ a $85 \%$ do total dos custos de um produto, durante todo seu ciclo de vida, são determinados nos estágios iniciais do seu projeto (Kaplan \& Cooper, 1998; Silva, 2001), derivando daí um dos aspectos que justificam a importância do DP no contexto organizacional.

Para Torres \& Miyake (2003), o DP pode ser entendido como um processo essencialmente intensivo em decisões. Esses autores destacam que diversos trabalhos e estudos têm sido desenvolvidos sobre a gestão do DP, tendo em vista o pensamento corrente e amplamente aceito de que o DP é central para o esforço de criação e sustentação da vantagem competitiva. Porém, apesar desta reconhecida importância creditada ao DP, a partir da análise da literatura sobre o tema, eles observam que em muitas empresas predominam ações do tipo 'apagar incêndios', ou seja, ações caracterizadas pela alocação não prevista de projetistas e/ou outros recursos para consertar problemas descobertos tardiamente no desenvolvimento.

Clark \& Wheelwright (1993) apontam para a existência de muitos elementos necessários para o sucesso da gestão do DP, entre os quais podem ser destacados: o acesso à tecnologia, a compreensão dos requisitos do cliente, conhecimento e especialização sobre os conceitos-chave do produto. Os autores destacam que, por si só, estes elementos não são suficientes para se obter excelência no DP, daí a necessidade de se promover a integração com as demais áreas funcionais da organização como forma de potencializar seus resultados. Dekkers et al. (2013) corroboram o ponto de vista sobre a necessidade de integração, destacando ainda o fato de que isto traz desafios significativos para diversas organizações industriais.

O PCP é uma das atividades da Administração da Produção, que consiste no estabelecimento de um plano operacional, preocupando-se em gerenciar as atividades da operação produtiva de modo a satisfazer a demanda dos consumidores operando continuamente (Slack et al., 2002). De outro modo, Burbidge (1981, p. 21) expressa que o PCP “[...] é a função da administração relacionada com o planejamento, direção e controle do suprimento de materiais e das atividades de processo em uma empresa."

Para Vollmann et al. (1997), o PCP provê informações para a administração eficiente do fluxo de materiais, para a efetiva utilização das pessoas e equipamentos disponíveis, para a coordenação das atividades internas com a dos fornecedores externos e para uma comunicação efetiva entre as necessidades do mercado consumidor e o sistema produtivo.

Uma representação comum do PCP inclui as seguintes características (Bonney, 2000):

a) uma hierarquia de planos detalhados progressivamente;

b) comunicação adequada, permitindo que os planos alcancem as pessoas devidas no momento oportuno;

c) feedback que fornece informações sobre o desempenho das atividades, possibilitando o acompanhamento dos planos e a tomada de ações corretivas.

Para Bonney (2000), o PCP é uma atividade bastante complexa em virtude das diversas incertezas e elementos inerentes às operações de manufatura (questões relacionadas à qualidade das entradas do Sistema de Produção; aspectos de manufaturabilidade dos produtos - complexidade dos projetos; layout fabril; incertezas associadas à demanda, entre outros).

Ao se estudar as atividades do PCP é comum sua segmentação em atividades relacionadas ao Planejamento da Produção (PP) e ao Controle da Produção (CP). Nesta perspectiva, o objetivo principal do PP é conseguir a compatibilização entre demanda e capacidade no médio prazo (geralmente entre 3 meses e 2 anos), enquanto que o objetivo principal do CP é regular o fluxo de materiais no curto prazo (Fernandes \& Godinho, 2010).

A partir da análise do trabalho de alguns autores (Burbidge, 1990; Vollmann et al. 1997; Slack et al., 2002; Fernandes \& Godinho, 2010), observa-se que independentemente do sistema produtivo, da tecnologia empregada no processo e da forma empregada para administrar a produção, existem algumas atividades que são inerentes à realização do PCP. Estas atividades são apresentadas no Quadro 1, já elencadas de acordo com sua alocação no PP e CP.

Outro aspecto que se destaca em relação ao PCP está relacionado ao seu elevado número de interfaces com as demais áreas funcionais para consecução de suas atividades, conforme mostra a Figura 2. Como exemplo destas interfaces, pode citar-se a interação com a área de Vendas para obtenção de informações que subsidiam o processo de PP.

Bonney (2000) destaca que as companhias precisam planejar e coordenar todos os estágios funcionais relacionados à disponibilização de um produto desde as etapas iniciais do projeto do produto, passando pela produção, distribuição e serviços pós-venda, atentando ainda para a necessidade da escolha 
Quadro 1. Atividades do PCP.

\begin{tabular}{|l|l|}
\hline PLANEJAMENTO DA PRODUÇÃO (PP) & CONTROLE DA PRODUÇÃO (CP) \\
\hline$\checkmark$ Previsão de demanda & $\checkmark$ Programação mestre \\
$\checkmark$ Planejamento agregado & $\checkmark$ Análise de capacidade \\
$\checkmark$ Planejamento da capacidade & $\checkmark$ Coordenação de ordens de compra e produção \\
$\checkmark$ Planejamento desagregado & $\checkmark$ Controle de estoques \\
& $\checkmark$ Controle de chão de fábrica \\
& o Liberação de ordens \\
& o Programação de operações \\
& o Apontamento de produção \\
\hline
\end{tabular}

Fonte: Elaborado a partir de Burbidge (1990); Vollmann et al. (1997); Slack et al. (2002); Fernandes \& Godinho (2010).

\section{Clientes}

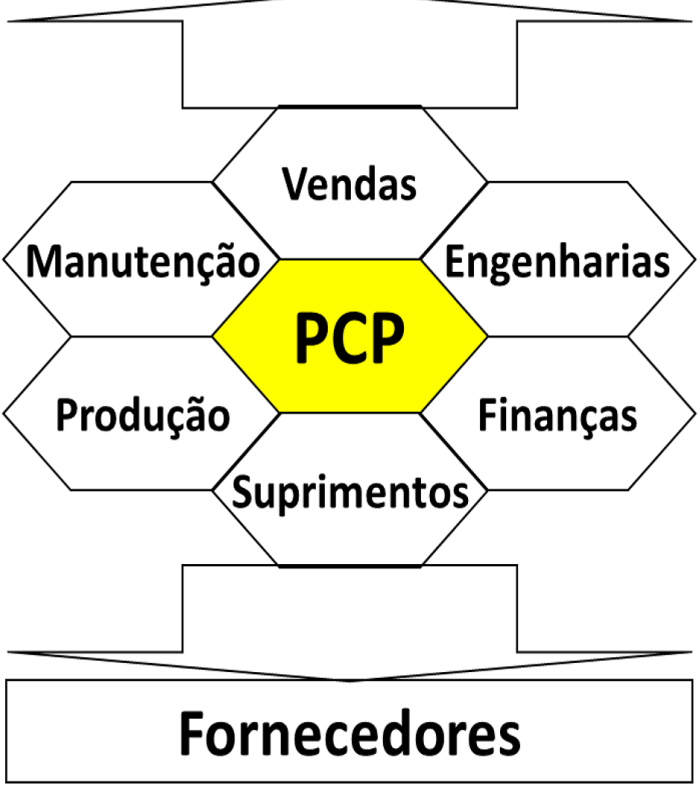

Figura 2. Interfaces do PCP com algumas áreas funcionais. Fonte: Elaborada a partir de diversas fontes entre elas Côrrea et al. (2010).

de sistemas que auxiliem esses estágios a serem planejados, coordenados e controlados.

De acordo com Davenport (1994, p. 259), “[...] nada é mais importante para o sucesso competitivo de uma empresa do que sua capacidade de desenvolver novos produtos e serviços, e entregá-los aos clientes [...]," salientando-se a necessidade de configuração adequada do Sistema de Produção de modo a alinhar-se para o atendimento dos requisitos de mercado.

Com relação a este alinhamento, destacam-se as Estratégias de Resposta à Demanda (ERD). Segundo Marçola (2000, p. 24), a ERD “[...] define como a manufatura irá responder à demanda, especialmente em termos temporal." Este autor salienta que cada categoria de ERD deve ser aplicada a produtos e situações específicas e para ter sucesso deve estar apropriadamente combinada com as características dos produtos e aspectos de competitividade temporal do mercado.

Uma classificação bastante detalhada das ERD pode ser encontrada em Fernandes \& Godinho (2010), os quais apresentam seis ERD que podem ser adotadas em um Sistema de Produção, as quais são:

- Produção para estoque com base em previsão de demanda (Make to Stock - MTS);

- Produção para estoque com base em uma rápida reposição de estoque (Quick response to stock-QRTS);

- Montagem sob Encomenda (Assemble to Order-ATO);

- Produção sob Encomenda (Make to Order-MTO);

- Recursos insumos sob encomenda (Resources to Order - RTO);

- Engenharia/Projeto sob Encomenda (Engineering to Order - ETO).

Pires (1995) destaca o fato de que com a adoção da ERD ETO os produtos são altamente customizados e o nível de interação com os clientes é muito grande. Moreira (1993, p. 12) observa que em processos que atendem à demanda por encomenda, "[...] as operações são ligadas a um cliente em particular com o qual se discute o preço e o prazo de entrega", nesta situação, uma medida-chave de desempenho é o prazo de entrega.

Fernandes \& Godinho (2010) observam que o tempo de resposta (tempo que o cliente vai esperar entre colocar um pedido e recebê-lo) é função dos lead times, que, por sua vez, são determinados de acordo com a ERD adotada. Por exemplo, se um Sistema de Produção adota a ERD ETO, seu tempo de resposta será uma soma dos lead times de projeto, de suprimento de materiais, de fabricação de componentes, montagem e distribuição. Enquanto que, adotando a ERD MTS, o tempo de resposta é 
composto apenas do lead time de distribuição. Outras características das empresas que adotam a ERD ETO são apresentadas na seção 2.3 .

\subsection{Integração interfuncional}

Segundo Vernadat (1996), integração significa o agrupamento de componentes heterogêneos de modo a formar um todo sinérgico e mais especificamente, integração empresarial é definida por esse autor como a preocupação em facilitar o fluxo de informações, materiais e o controle, ultrapassando as fronteiras organizacionais e conectando todas as funções necessárias, assim como suas entidades funcionais. $\mathrm{O}$ autor destaca que o propósito da integração no campo empresarial é melhorar a comunicação, a cooperação e a coordenação, promovendo ganhos de produtividade, flexibilidade e maior capacidade de gestão em ambientes de mudança.

A questão da integração e sua necessidade tem sido discutida no contexto das organizações há algum tempo e teve um forte impulso na década de 1990 com o advento das ideias da reengenharia de processos e a evolução da tecnologia da informação, inseridos no contexto de intensificação da competição no ambiente empresarial. De acordo com Oztemel \& Tekez (2009), as organizações precisam cada vez mais de habilidade para responder às rápidas mudanças do mercado e, para tanto, precisam modificar sua cultura e estrutura organizacional de modo a integrar e coordenar seus diferentes componentes.

Segundo Hvolby \& Trienekens (2010), nas últimas décadas foram observadas grandes mudanças no ambiente de negócio de diversas indústrias, ocasionadas tanto pelo desenvolvimento tecnológico, quanto pelo grande aumento na capacidade de comunicação, as quais intensificaram a competição em escala mundial. Para atuar em tal cenário, os autores salientam a necessidade de as empresas serem mais responsivas e desenvolverem processos mais enxutos e eficientes por meio da adaptação e integração das funções de negócio.

Na década de 1940, iniciavam-se na literatura administrativa alguns estudos que reconheciam a necessidade de um olhar mais abrangente para as organizações. Esses estudos chamavam a atenção para o fato de que as organizações são constituídas de partes inter-relacionadas que, conjuntamente, afetam o resultado global; dava-se início a 'Era dos Sistemas' (Churchman, 1972; Bertalanffy, 1977; Checkland, 1993). Diversos estudos, apesar de ainda não usarem o termo, tratavam da necessidade de integração entre os elementos que constituem um sistema.

Os benefícios advindos da integração das atividades no contexto organizacional são vários, entre eles: melhoria da comunicação; redução do tempo de atendimento de pedidos; aumento da qualidade; planejamento mais bem embasado devido ao compartilhamento de dados/informações; e ações de controle mais proativas em função da cooperação, coordenação e envolvimento na realização de atividades (Shapiro, 1977; Davenport, 1994; Vernadat, 1996; Song et al., 1998; Paiva et al., 2011; Sousa \& Lengler, 2011).

Questões pertinentes à integração têm sido bastante discutidas em diversos trabalhos notadamente sobre:

i) Tecnologia da informação, essencialmente no que tange à implantação e uso de sistemas ERP (Enterprise Resources Planning) para o compartilhamento de informações, melhoria e integração dos processos empresariais (Davenport, 1998; Souza, 2000; Manetti, 2001; Rondeau \& Litteral, 2001; Souza, 2005);

ii) Sobre o Processo de Desenvolvimento de Produtos (PDP) e sua Gestão, envolvendo a participação de departamentos, fornecedores e clientes no DP das empresas (Amaral \& Toledo, 2000; Tonioli, 2003; Mano \& Toledo, 2005; Onoyama, 2006; Onoyama et al., 2008; González, 2010; Chen et al., 2013);

iii) Em estudos relacionados à Gestão da Cadeia de Suprimentos, envolvendo incentivos à integração entre os elos da cadeia de suprimentos e agentes internos das organizações de modo a alcançar melhores resultados nos objetivos empresariais (Lambert et al., 1998; Croxton et al., 2001; Alvarez \& Queiroz, 2003; Smimova et al., 2011; Wu et al., 2013);

iv) Sobre os benefícios advindos da integração entre as funções do negócio - Marketing, Produção, P\&D (Pesquisa e Desenvolvimento), entre outras - no contexto da melhoria de desempenho organizacional (Shapiro, 1977; Griffin \& Hauser, 1996; Kahn, 1996; Kahn \& McDonough, 1997; Altamirano, 1999; Buss, 2002).

Lawrence \& Lorsch (1973) salientam dois importantes aspectos para o funcionamento das organizações quando consideradas como um sistema:

1. À medida que os sistemas crescem de tamanho, diferenciam-se em partes e o funcionamento destas partes separadas deve ser integrado para que o sistema inteiro seja viável;

2. A adaptação ao que se passa no mundo exterior destaca-se como uma importante função de qualquer sistema. 
Em relação à integração, Fernandes (1991, p. 21) salienta que "[...] empresas não integradas tendem a ser fortes em aspectos não tão essenciais e fracas em aspectos cruciais para as suas características de relacionamento com o mercado consumidor." Deste modo, a integração é fundamental para que uma empresa possa dosar os elementos essenciais para o negócio e consequentemente para a consecução dos objetivos organizacionais.

De acordo com Jugend (2010, p. 31), “[...] na área de gestão de operações, o tema integração possui dois enfoques distintos: da área de tecnologia da informação e da organização do trabalho." O enfoque da tecnologia de informação aborda a questão da integração a partir de mecanismos computacionais que integram diferentes departamentos e processos, enquanto o da organização do trabalho busca formas de aumentar a qualidade da colaboração existente entre os departamentos necessários para a realização dos trabalhos.

Davenport (1994, p. 272), ao tratar dos processos de manufatura, observa que "[...] na fabricação, como em outras funções, a melhor maneira de realizar os objetivos de tempo, serviços e flexibilidade é por meio de uma integração funcional cada vez maior." Isto se dá em função do potencial gerado pela combinação de capacidades das diversas áreas da organização. Há empresas que pretendem entregar produtos customizados em curto espaço de tempo; Davenport (1994) destaca que essas empresas têm que integrar rigorosamente as áreas de vendas, fabricação e logística num único processo de funcionamento perfeito e que, embora a tecnologia seja aplicada em vários pontos, ela se encontra subordinada à integração organizacional como um habilitador da mudança.

Ao tratar do assunto integração, a literatura destaca a existência de dois tipos possíveis: Integração Interorganizacional e Integração Intraorganizacional (também conhecida como Interdepartamental ou Interfuncional).

A integração Interorganizacional refere-se à colaboração estabelecida com clientes, fornecedores, detentores de tecnologia e institutos de pesquisa-externos à organização. Já a integração Intraorganizacional está associada ao compartilhamento de informações e envolvimento entre as funções ou departamentos internos da organização (Clark \& Wheelwright, 1993; Kahn, 1996; Sobral, 2003; Onoyama et al., 2008). Além disso, cada um desses dois tipos pode apresentar a perspectiva isolada ou a integrada. $\mathrm{Na}$ isolada, cada agente cuida somente de seus problemas e, na integrada, busca-se uma cooperação entre os agentes, de modo que os problemas de todos sejam melhor resolvidos.

De acordo com Kahn (1996), ao se revisar a literatura sobre integração interdepartamental, três correntes de pensamento podem ser identificadas. A primeira caracteriza a integração interdepartamental como simplesmente a interação ou comunicação relativa às atividades desenvolvidas entre os departamentos. Para a segunda, a caracterização se dá em função da colaboração existente entre os departamentos no desenvolvimento de suas atividades conjuntas. A terceira corrente adota uma perspectiva multidimensional, caracterizando a integração interdepartamental em função de ambas - interação e colaboração - indicando que deve haver o compartilhamento de informações e o envolvimento entre os departamentos.

Kahn (1996) define interação como algo que representa a natureza estrutural das atividades interdepartamentais, algo que coordena as atividades entre os departamentos. Envolve a realização de reuniões de rotina, teleconferências, o fluxo de documentos padrões, assim como o compartilhamento de informações. Já a colaboração é definida como algo não estruturado, envolvendo aspectos afetivos, dependente da vontade, da existência de processos comuns e compartilhados, entendimento e visão comuns, recursos e objetivos compartilhados. $\mathrm{O}$ autor salienta, ainda, que uma distinção complementar reside no fato de que a interação pode ser quantificada e controlada, enquanto a colaboração é algo mais intangível e difícil de controlar (Kahn, 1996).

$\mathrm{Na}$ abordagem que caracteriza a integração privilegiando os aspectos da interação, enfatiza-se o uso da comunicação na forma de reuniões e fluxos de informações entre os departamentos como forma de promovê-la. Nesta perspectiva, considera-se a comunicação como componente-chave da integração interdepartamental por meio de mais reuniões e melhores informações promovendo a relação interdepartamental (Kahn, 1996).

Já a abordagem que caracteriza a integração privilegiando os aspectos da colaboração enfatiza a execução coletiva do trabalho envolvendo o compartilhamento de objetivos como forma de promover a integração interdepartamental (Kahn, 1996).

Alguns parâmetros para se mensurar o nível de integração interdepartamental existente são: nível de comunicação, quantidade de esforços conjuntos, compartilhamento de informações e o nível de envolvimento entre as funções (Kahn, 1996). A questão central que deriva desta discussão é sobre como promover a integração entre as áreas funcionais do negócio.

Para Cardoso \& Miyake (2004), a análise da interface entre as funções é de fundamental importância para que as responsabilidades sejam definidas e os conhecimentos direcionados de modo a promover a integração funcional. Os autores sugerem que a reorganização da empresa com o propósito de racionalizar os fluxos de informação e materiais é um passo importante para se promover a integração. 
Segundo Lawrence \& Lorsch (1973), os primeiros teóricos que estudaram os aspectos relacionados à diferenciação e integração nas organizações tinham a ideia de que a integração se realizasse mediante um processo inteiramente racional e mecânico, ou seja, se a tarefa total da organização fosse dividida de acordo com certos princípios, a integração seria atendida expedindo-se ordens ao longo da hierarquia administrativa. Porém, a partir de estudos, foi possível constatar que a efetiva integração se dá por meio da resolução de conflitos que surgem devido a diferentes pontos de vista sustentados por especialistas funcionais. Ou seja, o papel das pessoas é crucial no que tange à integração no contexto empresarial.

Lawrence \& Lorsch (1973) relatam ainda que, em muitas organizações, estabelecem-se comitês, equipes integrantes ou são designados integradores individuais para facilitar a colaboração entre departamentos funcionais. Outras formas para promover a integração que esses autores observaram foi o controle de rotina, os processos de fixação de um roteiro de trabalho e, em alguns casos, atividades integradoras são realizadas fora dos canais oficiais por administradores individuais. Turkulainen \& Ketokivi (2012) chamam a atenção para o fato de que existem aspectos contingenciais ao se relacionar integração interfuncional e desempenho organizacional, demandando maior conexão e aprofundamento em estudos teóricos e empíricos.

De acordo com Mano \& Toledo (2005), o fato de muitas empresas adotarem uma estrutura organizacional funcional, na qual a experiência das pessoas e suas carreiras estão enraizadas nas suas funções, converte-se em um desafio para que ocorra a integração interdepartamental. Além disso, esses autores salientam que esta integração é frequentemente limitada pelo grau de comunicação e coordenação das atividades conjuntas entre as funções.

Hvolby \& Trienekens (2010) destacam que para se obter os benefícios da integração faz-se necessária a combinação entre novas tecnologias, mudanças organizacionais e comportamentais. Segundo estes autores, alguns desafios precisam ser superados, dentre os quais: a falta de confiança e a indisposição para se compartilhar informações; a baixa qualidade dos dados e informações; a extrema segmentação funcional das atividades e funções provocando perda da visão do processo.

Hvolby \& Trienekens (2010) listam como elementos-chave para promover a integração o compartilhamento de informações, a coordenação dos fluxos de trabalho e o planejamento sincronizado. Para os autores, ambientes que consigam operacionalizar esses elementos-chave têm como benefícios respostas mais rápidas às demandas do mercado, otimização da utilização de capacidade, ganhos de eficiência e acuracidade, assim como menores custos.
Shapiro (1977), ao estudar a interação entre as funções de Marketing e Produção, identificou alguns motivos que levam à existência de conflitos entre as áreas, dificultando sua integração, entre eles: a) avaliação e reconhecimento entre as funções; b) complexidade inerente à interface; c) orientação e experiência dos profissionais; d) diferenças culturais. Para gerenciar e minimizar tais conflitos, esse autor recomenda a definição de políticas claras, mudança nos indicadores de desempenho e a promoção do relacionamento interfuncional.

Melo (1995) observa que quanto maior a empresa, mais difícil se torna o gerenciamento das interfaces funcionais, tendo em vista o aumento do número de pessoas envolvidas e das interações necessárias para condução das atividades.

Nesse sentido, é fundamental que as organizações identifiquem os processos e funções-chave organizacionais que alicerçam sua competitividade e ataquem-nos em busca de excelência, tendo em vista que são eles que suportam a estratégia e o desempenho da organização na esfera de mercado em que atua e a integração interfuncional é apresentada aqui como uma opção para o processo de melhoria e busca da excelência operacional.

\subsection{A indústria de bens de capital}

Strachman \& Avellar (2008) destacam que a indústria de bens de capital reúne um conjunto de fabricantes de máquinas e equipamentos responsável, em grande medida, pela capacidade de produção de outros bens. As empresas que compõem esse setor industrial cumprem um papel determinante na difusão de novas tecnologias e na dinamização do crescimento econômico devido ao forte relacionamento com a produção de outros setores.

Conforme Strachman \& Avellar (2008), o setor industrial de bens de capital pode ser caracterizado por sua heterogeneidade, tanto de tipos quanto de usos, por seus objetivos e pelos setores a que se destinam seus produtos (químico, petroquímico, metalúrgico, têxtil, de calçados, elétrico, de papel e celulose, de alimentos, etc.). Essa heterogeneidade também está presente nas condições competitivas de vários mercados e segmentos, com suas especificidades em relação ao número, tamanho e capacitações das firmas que deles participam, condições de importação e exportação, etc. Maia et al. (2010) corroboram a observação sobre a heterogeneidade desse setor industrial, dada a grande variedade de tipos, de usos e de finalidades dos produtos, assim como das condições competitivas do mercado relacionadas ao ritmo tecnológico.

No que se refere à relevância econômica da indústria de bens de capital, sua contribuição é da ordem de $12 \%$ do valor agregado total da indústria 
de transformação brasileira, salientando-se o fato de que esse setor industrial conta com condições bastante heterogêneas de eficiência produtiva e competitividade internacional (Nassif, 2007).

Segundo Nassif (2007), existe pouca controvérsia a respeito da importância da indústria de bens de capital como propulsora do desenvolvimento econômico de um país. Tanto em termos micro, quanto macroeconômicos, é reconhecida sua importância como criadora de capacidade produtiva e indutora de progresso técnico.

Nassif (2007) observa que uma classificação tradicional divide a indústria em segmentos que produzem bens de capital sob encomenda, cujas características de maior sofisticação tecnológica e design adaptado para o atendimento de necessidades específicas do usuário requerem elevados gastos em P\&D e na engenharia do produto; e em segmentos que produzem bens de capital seriados, cuja produção padronizada e em série, por estar sujeita a elevadas economias de escala, costuma exigir não apenas escalas mínimas eficientes bastante elevadas, como também vultosos gastos em P\&D e em engenharia de processo.

Hill (1995), ao fazer uma comparação entre a indústria de bens de capital e a indústria de bens de consumo, destaca uma característica particular deste setor, observada em seu processo de negócio. Enquanto na indústria de bens de consumo segue-se a sequência engenharia-mercado-fabricação-venda, na indústria de bens de capital, a sequência mais comum é caracterizada por mercado-vendas-engenhariafabricação-instalação-operação e manutenção dos equipamentos.

Os trabalhos que estudam a indústria de bens de capital sob encomenda apontam para algumas peculiaridades dessas empresas quando da aplicação de conceitos e técnicas pré-existentes na teoria, demandando alguma adequação para a realidade dos processos e atividades dessas empresas. A exemplo dessas adequações, Rozenfeld et al. (2006, p. 508), ao discutir aspectos relacionados à aplicação de seu modelo de referência para o PDP, observam que para o caso de empresas que fabricam bens de capital com estratégia de resposta à demanda ETO, algumas 'mudanças profundas' são necessárias em fases do modelo.

Outra classificação também encontrada na literatura para as indústrias que fabricam bens de capital sob encomenda é a chamada One-of-a-Kind Production (OKP), caracterizando sua alta variedade de produtos, com pronunciada customização devido a requisitos específicos de clientes (Tu, 1997; Hameri \& Nihtilä, 1998; Tu et al., 2000; Pereira, 2005).

Avellar (2008) destaca a existência de poucos estudos acadêmicos sobre o setor de bens de capital, ressaltando a importância da realização de estudos que contemplem as características e necessidades destas empresas.

\section{Estudo de casos}

Conforme destacado na seção 1 , o método utilizado para obtenção dos dados nas empresas estudadas foi o estudo de caso. Yin (2005, p. 32) define o estudo de caso como "[...] uma investigação empírica que investiga um fenômeno contemporâneo dentro de um contexto da vida real, especialmente quando os limites entre o fenômeno e o contexto não estão claramente definidos". Tal tipo de procedimento envolve o estudo profundo e exaustivo de um ou poucos objetos de maneira que permita o seu amplo e detalhado conhecimento. Voss et al. (2002) destacam o estudo de caso como uma oportunidade para enriquecimento da teoria e possibilitam o desenvolvimento do pesquisador, porém observam que existem vários desafios ao conduzi-los, entre eles: o tempo consumido; a necessidade de habilidade para se entrevistar; e o cuidado ao se generalizar conclusões a partir de um limitado número de casos. Os pontos fortes desse método de pesquisa, apresentados por Yin (2005) e Voss et al. (2002), e o propósito do presente trabalho (explorar a integração entre DP e PCP de modo detalhado no ambiente em que o fenômeno ocorre), justificam a adoção desse método de pesquisa.

\subsection{Apresentação das empresas objeto de estudo}

A fim de preservar a identificação das empresas estudadas, estas serão denominadas como empresas A, $\mathrm{B}$ e C. A seleção dessas empresas se deu previamente com base na experiência profissional do pesquisador no setor industrial estudado, a qual foi fundamentada posteriormente com base em pesquisa bibliográfica e levantamento de informações sobre as empresas do Estado de São Paulo com pronunciada atuação no fornecimento de bens de capital por meio da ERD ETO. Neste sentido, trata-se de uma amostra intencional e não probabilística.

A representatividade das empresas $\mathrm{B}$ e $\mathrm{C}$, por exemplo, é apontada no trabalho de Avellar (2008), o qual as destaca em vista dos critérios: receita líquida anual (maior que R $\$ 100$ milhões), número de patentes no País e taxa de crescimento anual. A empresa A, apesar de não constar dos apontamentos do estudo de Avellar (2008), apresenta notório crescimento e participação no mercado, além de ter sido listada entre as dez maiores empresas do setor de bens de capital que atuam no Brasil pela revista Exame em 2011.

O Quadro 2 mostra um resumo dos critérios principais que levaram à seleção das empresas estudadas.

A empresa A foi fundada em 1920 e tem atuação histórica no fornecimento de bens de capital para o setor sucroalcooleiro. Na década de 1990 a empresa 
Quadro 2. Critérios principais adotados para seleção das empresas objeto de estudo.

\begin{tabular}{|c|c|c|}
\hline \multicolumn{2}{|c|}{ Critério } & Motivo \\
\hline Produto & Bens de Capital & Escopo da pesquisa \\
\hline Atividades-chave & Projeto e Fabricação & Possuir DP e PCP \\
\hline Porte & Grande $(>500$ funcionários $)$ & Maior complexidade da estrutura organizacional \\
\hline ERD predominante & ETO & Escopo da pesquisa \\
\hline Localização geográfica & Estado de São Paulo & Facilitar o acesso para condução dos estudos \\
\hline
\end{tabular}

Fonte: Elaboração própria.

realizou uma diversificação dos setores de mercado atendidos, passando a fornecer equipamentos para os setores siderúrgicos, mineração, alimentício, químico, petroquímico, de geração de energia, óleo e gás. Esta diversificação se deu devido a fusões e parcerias com alguns outros grupos empresariais, porém o fornecimento de equipamentos para o setor sucroalcooleiro continuou representando parcela significativa do volume de negócios da empresa.

A empresa A possui um parque fabril composto por nove unidades produtivas (sete no interior de São Paulo e duas na região nordeste), além de escritórios administrativos e comerciais distribuídos pelo Brasil. Emprega um total aproximado de 4000 funcionários, contando, também, com uma ampla cadeia de fornecedores e subfornecedores.

A empresa B foi fundada em 1953 e possui um histórico marcado por diversas parcerias e fusões com vários grupos empresariais, aumentando a gama de setores industriais atendidos e a diversidade de produtos comercializados. Exemplos de setores industriais atendidos pela empresa B incluem o petroquímico, hidrogeração, transporte, químico, siderúrgico e elétrico.

A empresa B emprega cerca de 5500 colaboradores alocados na fábrica, escritórios e em obras, abrangendo um escopo de atividades que vai além do projeto e fabricação de bens de capital sob encomenda. Sua principal planta industrial está localizada no interior do Estado de São Paulo, nela trabalham 2200 colaboradores focados nas atividades relacionadas ao projeto, fabricação e comercialização de bens de capital sob encomenda.

A empresa C foi fundada em 1911 e também tem como característica marcante o atendimento de diversos setores industriais e elevada variedade de produtos. Entre os setores atendidos, podem ser destacados o petroquímico, químico, hidrogeração, mineração e siderúrgico. Exemplos de equipamentos fornecidos por essa empresa compreendem desde aqueles relacionados à movimentação de materiais (pontes rolantes, esteiras transportadoras de materiais) até os mais diversos equipamentos empregados em processos industriais (vasos de pressão, equipamentos para processamento de minérios entre outros). O histórico dessa empresa também demonstra uma série de incorporações, parcerias e fusões com outros grupos empresariais. A empresa $\mathrm{C}$ conta com uma equipe de aproximadamente 2000 colaboradores, distribuídos em duas plantas industriais localizadas no Estado de São Paulo, sendo uma no interior do estado e outra na capital.

No que se refere à estrutura organizacional, as empresas $\mathrm{A}$ e $\mathrm{C}$ adotam uma estrutura funcional e a empresa $\mathrm{B}$ adota uma estrutura mista (funcional para algumas áreas e agrupamento de determinadas funções de negócio sob o comando da mesma gerência para outras).

\subsection{Análise da Integração entre DP e PCP nas empresas estudadas}

A fim de delinear o contexto no qual DP e PCP se inserem no ambiente ETO das empresas estudadas, um esboço do processo de atendimento de pedidos foi elaborado. A Figura 3 mostra uma visão geral desse processo, destacando as principais áreas envolvidas e atividades realizadas. A fim de ilustrar o processo de atendimento de um pedido, optou-se por sua segmentação em quatro fases (Negociar Venda, Especificar Produto, Planejar Materiais e Planejar Fabricação e Entrega). A segmentação do processo de atendimento de pedido conforme apresentada na Figura 3 foi realizada pelo fato de serem macrofases bem definidas no processo de atendimento de pedido, nas quais DP e PCP obtêm e geram dados que auxiliam na consecução dos objetivos do atendimento de pedido. $\mathrm{O}$ embasamento para tal foi a experiência do pesquisador (atuação profissional desde $2007 \mathrm{em}$ empresas desse segmento) e observação detalhada (possibilitada pelos estudos de casos nas empresas A, B e C). Trata-se de um processo geral para as três empresas. A delimitação ao processo de atendimento de pedidos se faz válida por contemplar todo o escopo de atividades no qual DP e PCP se inserem, vale lembrar que são empresas que trabalham sob encomenda, ou seja, atender pedido é a essência do negócio, além disso, é o processo no qual devem ser focadas as ações de melhoria para busca da excelência operacional.

O início do atendimento de um pedido se dá por meio do levantamento de dados sobre as necessidades do cliente. Nesse momento, a área comercial faz uma primeira abordagem com o cliente a fim de obter os 


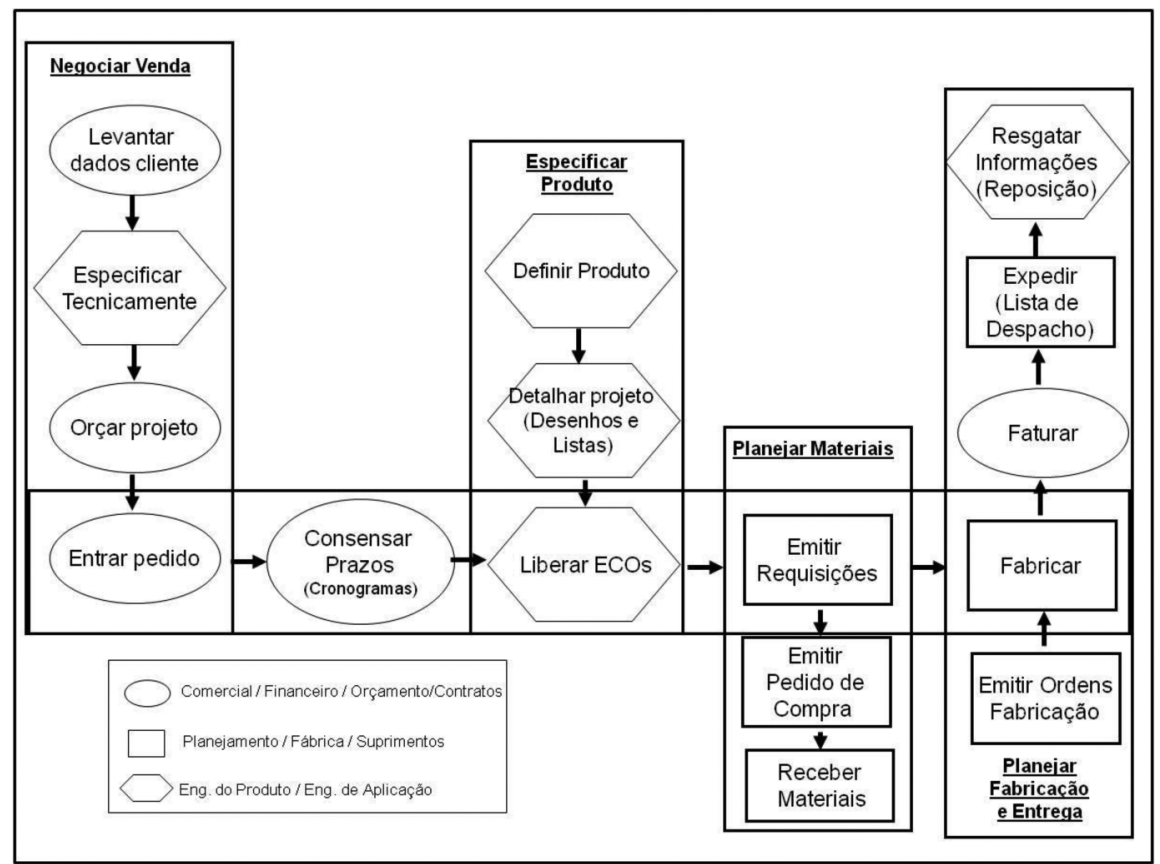

Figura 3. Principais áreas e atividades do processo 'atender pedido'. Fonte: Elaboração própria.

dados técnicos e dar início às negociações comerciais a respeito do fornecimento. Esses dados preliminares são tratados por uma Engenharia de Aplicação, a fim de desenvolver uma Proposta Técnica (PT) detalhada sobre o equipamento a ser fornecido - neste momento, diversas interações são realizadas com o cliente a fim de refinar e obter dados que permitam a correta especificação do produto.

Concluída a etapa de elaboração da PT pela área de Engenharia de Aplicação, é encaminhada para a área de Orçamentos, a fim de que os custos envolvidos no fornecimento sejam estimados e possibilitem que a área comercial forme o preço de venda. Importante destacar que, na etapa de orçamentação, diversas áreas da empresa são consultadas a fim de fornecer dados e informações que possibilitem maior precisão nas estimativas a serem apresentadas. Exemplos a este respeito são caracterizados pela interação com Suprimentos a fim de realizar consultas a fornecedores da Engenharia Industrial ou de Processos sobre tempos e métodos a serem empregados para fabricação dos equipamentos. Nesta fase, o PCP também é consultado pela área comercial sobre estimativas de prazos de entrega. Além disto, diversas informações de histórico de fornecimento também são utilizadas, destacando a importância do compartilhamento de informações.

Concluída a etapa de orçamentação e com a PT finalizada, a área Comercial negocia a venda com o cliente e, a partir do momento de sua efetivação, é realizada a entrada do pedido na empresa, momento no qual as demais áreas são oficialmente acionadas para dar início ao processo de fornecimento do equipamento. Nessa ocasião, uma cópia da PT é encaminhada pela área Comercial para as áreas de Administração de Contratos, PCP, Suprimentos e Engenharia do Produto.

Além da PT, é disponibilizada também uma cópia do pedido interno de vendas. Essa disponibilização inicial de informações é realizada a fim de que os envolvidos tomem conhecimento das condições negociadas com o cliente no que se refere a requisitos técnicos do produto, prazos e condições de entrega.

É comum nesse momento que as áreas designem responsáveis para cuidar do processo de fornecimento em questão, ficando esse como responsável por cuidar dos assuntos pertinentes àquele fornecimento no que se refere ao escopo de atuação da sua área funcional. Cada fornecimento é tratado como um projeto e estas pessoas são designadas como membros da equipe de projeto, porém de modo informal.

Uma das primeiras atribuições desta equipe de projeto é obter o consenso de prazos, que consiste basicamente na definição e validação do cronograma de atividades para entrega do equipamento e discussão das informações previamente disponibilizadas, envolvendo o esclarecimento de dúvidas e possíveis sugestões para o correto atendimento dos requisitos especificados.

Depois da conclusão do cronograma de fornecimento, a Engenharia do Produto realiza a especificação do produto. Partindo dos dados iniciais disponíveis na PT e análises de engenharia, o produto é definido tecnicamente resultando na elaboração de desenhos, listas e especificações de materiais. Estas informações são liberadas para a área de PCP, por meio das 
Engineering Change Orders (ECO) ou Ordens de Alteração de Engenharia.

A partir do momento em que as listas de materiais e desenhos são recebidas na área de PCP, inicia-se o processo de planejamento de materiais. Este processo envolve a emissão de requisições para aprovisionamento de materiais disponíveis em estoque e requisições de compra para materiais não disponíveis. Quando da emissão de requisições de compra, estas são encaminhadas para área de Suprimentos, a fim de que fornecedores sejam acionados, para o suprimento de tais necessidades em termos de especificação dos materiais, quantidades e prazos indicados nas requisições oriundas do PCP. Este processo se encerra na efetivação do recebimento dos materiais solicitados.

Concluída a etapa de planejamento de materiais, inicia-se o processo de planejamento da fabricação e entrega do produto. Nesse processo, além da área de PCP, atuam também as áreas de Engenharia Industrial/Processos (elaboração de croquis, roteiros e processos de fabricação) e Qualidade (elaboração de planos de inspeção e testes). Essencialmente ao PCP, cabe a emissão das Ordens de Fabricação e o detalhamento dos planos e programas de fabricação que orientarão a execução das atividades no chão de fábrica (atividades de médio e curto prazo).

A entrega do produto é tratada neste momento em virtude das elevadas dimensões dos equipamentos e do fato de que em muitas situações o produto é expedido em partes após a conclusão de etapas intermediárias de fabricação.

Pelo fato de se tratarem de produtos altamente customizados (projeto e fabricação sob encomenda), é de suma importância o registro de todas as informações e alterações de produto ocorridas ao longo deste processo, a fim de que, em fornecimentos futuros, sejam eles de equipamentos completos para o mesmo cliente ou prestação de serviços de manutenção e fornecimento de peças de reposição, estas informações possam ser resgatadas.

Apesar de esse processo ter sido apresentado de forma macro e sequencial, destaca-se que, no atendimento de pedido desse tipo de empresa, a quantidade de interações entre as áreas e o fluxo bidirecional de informações é elevado, intensificando a necessidade de comunicação eficiente e colaboração entre as áreas para que os requisitos do cliente e objetivos de desempenho da empresa sejam atingidos. Aspecto que exemplifica esta colocação pode ser demonstrado pela liberação em partes do projeto do produto, ou seja, à medida que a Engenharia do Produto conclui o projeto de uma parte do equipamento (conjuntos, subconjuntos ou até mesmo peças), estas são liberadas para que as providências de planejamento e fabricação sejam tomadas, havendo grande necessidade de coordenação das atividades, a fim de que retrabalhos e erros não ocorram.

\section{Barreiras e desafios para melhoria da Integração entre DP e PCP em ambiente ETO}

A fim de se identificar barreiras e desafios que precisam ser superados para melhoria da integração entre DP e PCP, quando da adoção da ERD ETO na fabricação de bens de capital sob encomenda, foram listados tanto problemas observados no processo de atendimento de pedidos das empresas estudadas quanto aspectos relacionados à estrutura organizacional e forma de interação entre as áreas.

No Quadro 3, são apresentados alguns problemas típicos, comuns às três empresas estudadas, destacando-se sua causa principal e seu impacto no processo de atendimento de pedidos.

Tais problemas foram obtidos por meio de observação direta do pesquisador (em período de atuação profissional), posteriormente foram relacionados e apresentados nas empresas A, B e C para profissionais que atuam no DP e no PCP, momento no qual esses profissionais respondem a um questionário com escala Likert, a fim de identificar o seu grau de concordância em relação aos problemas apresentados. A confirmação empírica preliminar desses problemas se deu devido ao alto grau de concordância obtida. Uma análise desses problemas demonstra sua relação direta com a falta de integração entre as áreas envolvidas, pois notadamente são verificadas deficiências relacionadas à interação (comunicação e compartilhamento de informações) e colaboração (pré-disposição para condução do trabalho conjuntamente e confiança mútua).

A Figura 4 mostra uma representação simplificada do organograma de uma empresa que adota uma estrutura funcional para a segmentação das funções empresariais (forma mais comum encontrada nas organizações e evidenciada nas empresas estudadas). Destaca-se o fluxo dos processos de atendimento, cruzando as diversas linhas funcionais e as interfaces entre as áreas, com a informação partindo do geral (requisitos do cliente), para o específico (detalhamento destes requisitos pelas áreas e geração do produto). Nestas circunstâncias, são observadas 'zonas de problemas' nas quais se manifestam os problemas relacionados à falta de integração. Esta figura é fruto da análise dos autores, tendo em vista que nas empresas estudadas predomina a estrutura funcional, o processo de atendimento de pedidos em ambiente ETO cruza diversas áreas funcionais (conforme mostrado na Figura 3) e os problemas de integração ocorrem, predominantemente, nas interfaces entre as áreas (conforme Quadro 3).

Exemplos observados nos casos estudados de situações ou aspectos que provocam deficiências de integração e manifestam-se nestas 'zonas de problemas' são: 
Quadro 3. Problemas típicos observados no processo ‘Atender Pedidos' em ambiente ETO.

\begin{tabular}{|c|c|c|c|}
\hline Item & Problema & Causa Principal & Impacto no Processo \\
\hline 01 & $\begin{array}{l}\text { Dados e informações iniciais sobre } \\
\text { requisitos do cliente incompletos ou } \\
\text { inconsistentes. }\end{array}$ & $\begin{array}{l}\text { Baixa interação entre comercial } \\
\text { e engenharias de aplicação e } \\
\text { produto. } \\
\text { Informações insuficientes por } \\
\text { parte do cliente neste momento. }\end{array}$ & $\begin{array}{l}\text { Atrasos e dificuldades para } \\
\text { concluir a PT. } \\
\text { Erros de especificação nas } \\
\text { etapas seguintes do processo. }\end{array}$ \\
\hline 02 & $\begin{array}{l}\text { Desvios entre o que está previsto na } \\
\text { PT e o que efetivamente foi projetado. }\end{array}$ & $\begin{array}{l}\text { Não cumprimento do que } \\
\text { está especificado na PT pela } \\
\text { engenharia ou correções } \\
\text { realizadas pela engenharia sem a } \\
\text { devida revisão da PT. }\end{array}$ & $\begin{array}{l}\text { Não atendimento do requisito } \\
\text { do cliente. } \\
\text { Aquisição e fabricação de } \\
\text { materiais desnecessários. } \\
\text { Aumento dos estoques. } \\
\text { Divergências de custos. }\end{array}$ \\
\hline 03 & $\begin{array}{l}\text { Desvios entre informação de projeto } \\
\text { e itens gerados na fabricação ou } \\
\text { compra. }\end{array}$ & $\begin{array}{l}\text { Falta de retorno de informações } \\
\text { para a Engenharia de Produto. }\end{array}$ & $\begin{array}{l}\text { Projeto em desacordo com } \\
\text { equipamento efetivamente } \\
\text { fornecido. }\end{array}$ \\
\hline 04 & $\begin{array}{l}\text { Atrasos em relação aos prazos } \\
\text { negociados em cronogramas. }\end{array}$ & $\begin{array}{l}\text { Falhas de coordenação e } \\
\text { comunicação entre as áreas. } \\
\text { Não utilização do cronograma } \\
\text { como ferramenta de gestão e } \\
\text { coordenação das atividades. }\end{array}$ & $\begin{array}{l}\text { Não cumprimento de prazos } \\
\text { acordados com cliente interno } \\
\text { e externo. }\end{array}$ \\
\hline 05 & $\begin{array}{l}\text { Elevado número de revisões de } \\
\text { projeto. }\end{array}$ & $\begin{array}{l}\text { Inconsistência nos dados e } \\
\text { informações previamente } \\
\text { disponibilizados. }\end{array}$ & $\begin{array}{l}\text { Retrabalho devido a alterações } \\
\text { em etapas avançadas de } \\
\text { execução. } \\
\text { Custos não previstos. } \\
\text { Atividades realizadas com } \\
\text { informações obsoletas. }\end{array}$ \\
\hline 06 & $\begin{array}{l}\text { Prazos reduzidos para consecução das } \\
\text { atividades. }\end{array}$ & $\begin{array}{l}\text { Pressuposto de que o prazo não } \\
\text { será cumprido. }\end{array}$ & $\begin{array}{l}\text { Pressão desnecessária e erros } \\
\text { nas atividades. }\end{array}$ \\
\hline
\end{tabular}

Fonte: Elaboração própria.

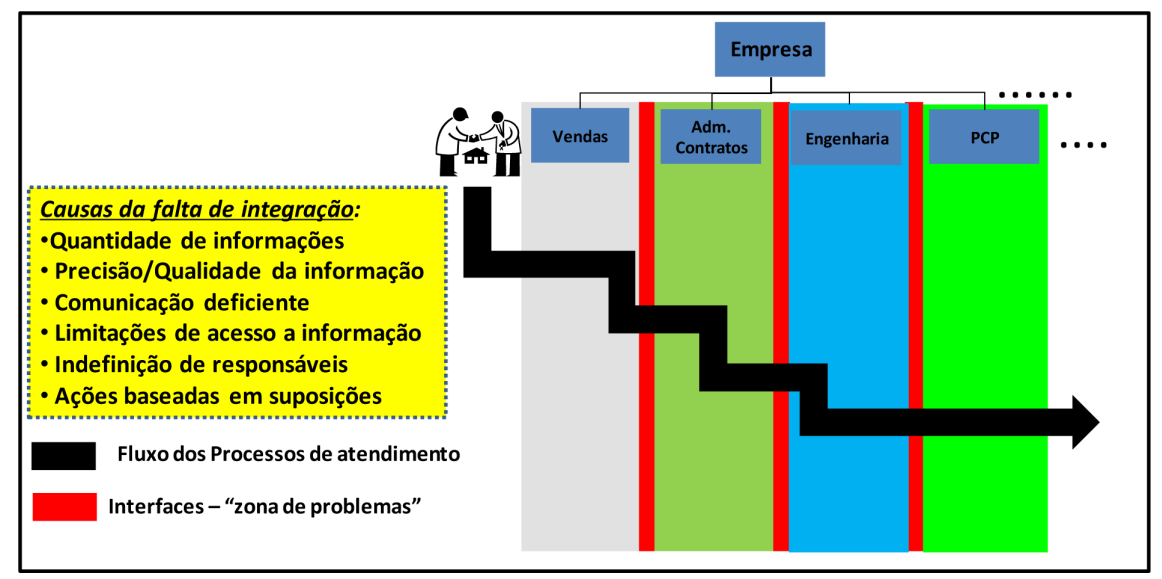

Figura 4. Troca de informações e causas da falta de integração no contexto das empresas estudadas. Fonte: Elaboração própria.

a) Elevada quantidade de informações - em processos que envolvem elevada troca de informações, seja pela complexidade dos produtos ou dos processos e atividades envolvidas, aumentando a incidência de problemas relacionados à comunicação e uso da informação.

b) Precisão/Qualidade da informação - baixa acuracidade das informações trocadas entre as áreas, seja por falta de feedback ou desconhecimento das etapas posteriores dos processos.

c) Comunicação deficiente ou limitações de acesso à informação - falta de mecanismos que simplifiquem ou eliminem ruídos dos processos de comunicação e/ou não compartilhamento de informações devido a resistências culturais (informação vista como forma de poder). 
d) Indefinição de responsáveis - responsabilidades mal atribuídas ou falta de acompanhamento e controle, gerando 'abandono' ou ruptura de processos nas interfaces entre as áreas, tendo em vista, também, que os processos de atendimento de pedido cruzam diversas linhas de comando funcionais.

e) Ações baseadas em suposições - desconhecimento das interligações e interdependências entre os processos e atividades das áreas, fazendo com que ações sejam tomadas com base em suposições de modo a gerar dados e informações não condizentes com as necessidades reais.

Essas barreiras e desafios têm impacto significativo nos resultados obtidos pelas empresas estudadas, necessitando de ações sistemáticas e consistentes para sua minimização. Nota-se que as relações interfuncionais afetam diretamente os aspectos referentes ao atendimento das necessidades do mercado (essencialmente pontualidade de entrega, custo e qualidade) e a melhoria da integração interfuncional é questão fundamental.

\section{Conclusões}

O objetivo do presente trabalho foi apresentar e discutir barreiras e desafios para a melhoria da integração entre DP e PCP em ambiente ETO. A literatura mostra, de maneira clara, os ganhos que podem ser obtidos com base na melhoria da integração interfuncional, destacando-se alguns caminhos para sua consecução no contexto organizacional (interação, colaboração ou ambas). Por outro lado, o papel do DP e do PCP é crucial para tais empresas. Porém, mesmo assim, após algumas décadas de discussões e pesquisas sobre o assunto integração, lacunas podem ser observadas no que se refere à sua utilização para obtenção da excelência operacional, como no caso das empresas estudadas.

É notório observar que um cenário efetivo de integração ainda não é observado nas empresas objeto de estudo, seja pela não aplicação consistente dos conceitos e práticas já existentes, seja por peculiaridades inerentes aos seus produtos, processos e atividades.

Destaca-se que a melhoria da integração não pode ser atingida simplesmente pela adoção e aplicação de ferramentas de tecnologia da informação - postura adotada por diversas empresas ao adquirirem sistemas informatizados na expectativa de que isso as torne mais integradas. Nesse sentido, são notórias a importância das pessoas e do entendimento e configuração das funções e processos-chave do Sistema de Produção como elementos preponderantes para a efetiva consecução da integração interfuncional. Além disto, destaca-se a necessidade de melhor explorar as inter-relações e interfaces entre os processos empresariais, buscando-se uma forma mais dinâmica e consistente de se produzir valor e atender aos objetivos de desempenho priorizados.

Por fim, vale ressaltar a necessidade de estudos que contemplem as particularidades e necessidades da indústria de bens de capital em ambiente ETO, a fim de indicar caminhos e ações para a efetiva integração entre DP e PCP, superando as barreiras e desafios observados. Em meio a essa discussão, uma contribuição do presente trabalho é explorar detalhadamente a questão da integração entre duas funções-chave organizacionais para o tipo de Sistema de Produção estudado, destacando processos, atividades e problemas práticos observados por meio do estudo aprofundado de três empresas com significativa representatividade no setor estudado. Outra contribuição, no que se refere ao estado da arte do tema estudado, diz respeito à indicação de lacunas entre teoria e prática, tendo em vista que foram apresentados problemas ainda não observados na literatura disponível (os quais demandariam outros estudos para comprovação - limitação do estudo de caso em generalizar resultados) e, não foram encontrados outros trabalhos que tratem especificamente da integração entre DP e PCP, notadamente em Sistemas de Produção que adotam a ERD ETO.

\section{Referências}

Altamirano, A. V. (1999). Metodologia para integração do marketing e da manufatura (Tese de doutorado). Universidade Federal de Santa Catarina, Florianópolis.

Alvarez, M. P., \& Queiroz, A. A. (2003). Aproximações dos laços de parcerias entre fornecedor-cliente na cadeia de suprimentos como fonte de competitividade. In XXIII Encontro Nacional de Engenharia de Produção. Ouro Preto.

Amaral, D. C. (2001). Arquitetura para gerenciamento de conhecimentos explícitos sobre o processo de desenvolvimento de produto (Tese de doutorado). EESC-USP, São Carlos.

Amaral, D. C., \& Toledo, J. C. (2000). Colaboração clientefornecedor no processo de desenvolvimento de produto: estudo de casos na indústria de autopeças. Gestão \& Produção, 7(1), 56-72. http://dx.doi.org/10.1590/S0104$530 \times 2000000100005$.

Avellar, A. P. (2008). Relatório setorial final: bens de capital. Pesquisa DPP-Diretório de Pesquisa Privada. Recuperado em março de 2010, de http://www.finep. gov.br/PortalDPP

Bertalanffy, L. (1977). Teoria geral dos sistemas (3. ed.). Petrópolis: Vozes.

Bonney, M. (2000). Reflections on production planning and control (PPC). Gestão e Produção, 7(3), 181-207. 
Burbidge, J. L. (1981). Planejamento e controle da produção. São Paulo: Atlas.

Burbidge, J. L. (1990). Production control: a universal conceptual framework. Production Planning and Control, 1(1), 3-16. http://dx.doi.org/10.1080/09537289008919289.

Buss, C. O. (2002). Cooperação interfuncional no desenvolvimento de novos produtos: a interface marketingengenharia (Dissertação de mestrado). Universidade Federal do Rio Grande do Sul, Porto Alegre.

Cardoso, A. A. C., \& Miyake, D. I. (2004). Integração entre as funções de produção e PCP pela promoção do sistema puxado. In XI Simpósio de Engenharia de Produção (SIMPEP). Bauru.

Checkland, P. B. (1993). Systems thinking, systems pratice (3th ed.). Chichester: John Wiley \& Sons.

Chen, Y. C., Li, P. C., \& Lin, Y. H. (2013). How interand intra-organisational coordination affect product development performance: the role of slack resources. Journal of Business and Industrial Marketing, 28(1-2), 125-136. http://dx.doi.org/10.1108/08858621311295263.

Churchman, C. W. (1972). Introdução à teoria dos sistemas (2. ed.). Petrópolis: Vozes.

Clark, K. B., \& Wheelwright, S. C. (1993). Managing new product and process development. New York: Free Press.

Corrêa, H. L., \& Gianesi, I. G. N. (2007). Just in time, MRP II e OPT: um enfoque estratégico (2. ed.). São Paulo: Atlas.

Corrêa, H. L., Gianesi, I. G. N., \& Caon, M. (2010). Planejamento, programação e controle da produção: MRP II/ERP - conceitos, uso e implantação (5. ed.). São Paulo: Atlas.

Croxton, K. L., García-Dastugue, S. J., Lambert, D. M., \& Rogers, D. S. (2001). The supply chain management processes. The International Journal of Logistics Management, 12(2), 13-36. http://dx.doi. org/10.1108/09574090110806271.

Davenport, T. H. (1994). Reengenharia de processos (5. ed.). Rio de Janeiro: Campus.

Davenport, T. H. (1998). Putting the enterprise into the enterprise system. Harvard Business Review, 76(4), 121-131. PMid:10181586.

Dekkers, R., Chang, C. M., \& Kreutzfeldt, J. (2013). The interface between "product design and engineering" and manufacturing: a review of the literature and empirical evidence. International Journal of Production Economics, 144(1), 316-333. http://dx.doi.org/10.1016/j. ijpe.2013.02.020.

Dilworth, J. B. (1993). Productions and Operations Management: manufacturing and services (5th ed.). New York: McGraw-Hill.

Fernandes, F. C. F. (1991). Concepção de um Sistema de Controle da Produção para a manufatura celular (Tese de doutorado). EESC-USP, São Carlos.
Fernandes, F. C. F., \& Godinho, M., Fo. (2010). Planejamento e controle da produção: dos fundamentos ao essencial. São Paulo: Atlas.

González, M. O. A. (2010). Processo de gerenciar a integração de clientes no processo de desenvolvimento de produtos (Tese de doutorado). Centro de Ciências Exatas e Tecnologia, Departamento de Engenharia de Produção, Universidade Federal de São Carlos, São Carlos.

Griffin, A., \& Hauser, J. R.(1996). D and marketing: a review and analysis of the literature. Journal of Product Innovation Management, 13(3), 191-215. http://dx.doi. org/10.1111/1540-5885.1330191.

Groover, M. P. (2011). Automação industrial e sistemas de manufatura (3. ed.). São Paulo: Pearson Prentice Hall.

Hameri, A., \& Nihtilä, J. (1998). Product data management: exploratory study on state-of-the-art in one-of-a-kind product industry. Computers in Industry, 35(3), 195206. http://dx.doi.org/10.1016/S0166-3615(98)00064-5.

Hayes, J. P., Pisano, G., Upton, D., \& Wheelwright, S. (2008). Produção, Estratégia e Tecnologia: em busca da vantagem competitiva. Porto Alegre: Bookman.

Hill, A. (1995). Competitiveness and processes: integrating engineering in the capital goods industry. World Class Design to Manufacture, 2(5), 27-31. http://dx.doi. org/10.1108/09642369310095193.

Hvolby, H. H., \& Trienekens, J. H. (2010). Challenges in business systems integration. Computers in Industry, 61(9), 808-812. http://dx.doi.org/10.1016/j. compind.2010.07.006.

Jugend, D. (2010). Gestão da integração entre desenvolvimento de produtos e de tecnologias: estudo de casos em empresas industriais de médio porte e intensivas em tecnologia (Tese de doutorado). Centro de Ciências Exatas e Tecnologia, Departamento de Engenharia de Produção, Universidade Federal de São Carlos, São Carlos.

Kahn, K. B. (1996). Interdepartmental Integration: a definition with implications for product development performance. Journal of Product Innovation Management, 13(2), 137150. http://dx.doi.org/10.1016/0737-6782(95)00110-7.

Kahn, K. B., \& McDonough, E. F. 3rd (1997). An empirical study of the relationships among co-location, Integration, performance, and satisfaction. Journal of Product Innovation Management, 14(3), 162-176. http://dx.doi. org/10.1111/1540-5885.1430161.

Kaplan, R. S., \& Cooper, R. (1998). Custo e desempenho: administre seus custos para ser mais competitivo. São Paulo: Futura.

Lambert, D. M., Cooper, M. C., \& Pagh, J. D. (1998). Supply chain management: implementation issues and research opportunities. The International Journal of Logistics Management, 9(2), 1-19. http://dx.doi. org/10.1108/09574099810805807. 
Lawrence, P. R., \& Lorsch, J. W. (1973). As empresas e o ambiente: diferenciação e integração administrativas. Petrópolis: Vozes.

Maia, G. T. O., Martins, R. A., \& Maia, J. L. (2010). Medição de desempenho em uma empresa de bens de capital: um estudo de caso. In Anais do XXX Encontro Nacional de Engenharia de Produção (ENEGEP). São Carlos.

Manetti, J. (2001). How technology is transforming manufacturing. Production and Inventory Management Journal, 42(1), 54-64.

Mano, A. P., \& Toledo, J. C. (2005). A integração interfuncional na gestão de desenvolvimento do produto: um estudo de caso em uma empresa de máquina agrícola. In Anais do V Congresso Brasileiro de Gestão do Desenvolvimento de Produtos. Curitiba.

Marçola, J. A. (2000). Horas anualizadas como técnica do planejamento de capacidade em sistemas de manufatura (Tese de doutorado). EESC-USP, São Carlos.

Melo, D. A. (1995). Discussão sobre a problemática na interface entre as funções marketing e produção. Produção, 5(1), 79-90.

Moreira, D. A. (1993). Administração da produção e operações. São Paulo: Pioneira Thomson Learning.

Nassif, A. (2007). Estrutura e competitividade da Indústria de bens de capital brasileira (Texto para Discussão, 109). Rio de Janeiro: BNDES.

Onoyama, S. S. (). Integração multifuncional no desenvolvimento de produtos: estudo de múltiplos casos em indústrias de laticínios mineiras (Dissertação de mestrado). UFMG, Belo Horizonte, 2006.

Onoyama, S. S., Onoyama, M. M., Lara, J. E., Assumpção, M. R. P., \& Toledo, J. C. (2008). Integração intra e interorganizacional no desenvolvimento de produtos: estudo de caso no setor de laticínios. Revista Gestão Industrial, 4(1), 68-87.

Oztemel, E., \& Tekez, E. K. (2009). Integrating manufacturing systems through knowledge exchange protocols within an agent-based knowledge network. Robotics and Computer-integrated Manufacturing, 25(1), 235-245. http://dx.doi.org/10.1016/j.rcim.2007.12.001.

Paiva, E. L., Gavronski, I., \& D’Avila, L. C. (2011). The relationship between manufacturing integration and performance from an activity-oriented perspective. Brazilian Administration Review, 8(4), 376-394.

Pereira, M. M. (2005) Avaliação de um ambiente computacional integrado para desenvolvimento de produtos no seguimento de bens de capital com engenharia sob encomenda (Dissertação de mestrado). EESC-USP, São Carlos.

Pires, S. R. I. (1995). Gestão estratégica da produção. Piracicaba: UNIMEP.

Rondeau, P. J., \& Litteral, L. A. (2001). Evolution of manufacturing planning and control systems: from reorder point to enterprise resource planning. Production an Inventory Management Journal, 42(2), 1-7.

Rozenfeld, H., Forcellini, F. A., Amaral, D. C., Toledo, J. C., Silva, S. L., Alliprandini, D. H., \& Scalice, R. K. (2006). Gestão de desenvolvimento de produtos: uma referência para melhoria do processo. São Paulo: Saraiva.

Shapiro, B. P. (1977). Can marketing and manufacturing co-exist? Harvard Business Review, 55, 104-114.

Silva, C. E. S. (2001). Método para avaliação do desempenho do processo de desenvolvimento de produto (Tese de doutorado). UFSC, Santa Catarina.

Sipper, D., \& Bulfin, R. (1997). Production: planning, control and integration. McGraw-Hill.

Slack, N., Chambers, S., Harland, C., Harrison, A., \& Johnston, R. (2002). Administração da produção (2. ed.). São Paulo: Atlas.

Smimova, M., Henneberg, S. C., Ashnai, B., Naude, P., \& Mouzas, S. (2011). Understanding the role of marketingpurchasing collaboration in industrial markets: the case of Russia. Industrial Marketing Management, 40(1), 5464. http://dx.doi.org/10.1016/j.indmarman.2010.09.010.

Sobral, M. C. (2003). Gestão do Desenvolvimento de Produtos: um contraste entre desenvolvimento conjunto e desenvolvimento interno (Dissertação de mestrado). FEA-USP, São Paulo.

Song, X. M., Thieme, R. J., \& Xie, J. (1998). The impact of cross-functional joint involvement across product development stages: an exploratory study. Journal of Product Innovation Management, 15(4), 289-302. http:// dx.doi.org/10.1016/S0737-6782(97)00108-2.

Sousa, C. M. P., \& Lengler, J. (2011). Examining the determinants of interfunctional coordination and export performance: an investigation of Brazilian exporters. International Marketing: Emerging Markets, 21, 189-206. http://dx.doi.org/10.1108/S1474-7979(2011)0000021011.

Souza, C. A. (2000). Sistemas integrados de gestão empresarial: estudos de casos de implantação de sistemas ERP (Dissertação de mestrado). FEA-USP, São Paulo.

Souza, L. C. (2005). O uso do ERP (Enterprise Resource Planning) e seu impacto na gestão de suprimentos em empresas da indústria de alimentos processados (Dissertação de mestrado). DEP-UFSCar, São Carlos.

Strachman, E., \& Avellar, A. P. M. (2008). Estratégias, desenvolvimento tecnológico e inovação no setor de bens de capital no Brasil. Ensaios FEE, 29(1), 237-266.

Tonioli, J. N. (2003). A integração entre o processo de desenvolvimento de produto e o gerenciamento da cadeia de suprimentos e sua relação com o papel desempenhado pelo engenheiro de produto (Dissertação de mestrado). Escola Politécnica-USP, São Paulo.

Torres, N. Jr., \& Miyake, D. I. (2003). A melhoria contínua no processo de desenvolvimento de produtos: identificando 
seus elementos e ocorrência. In IV Congresso Brasileiro de Gestão de Desenvolvimento de Produtos. Gramado.

Tu, Y. (1997). Production planning and control in a virtual one-of-a-kind production company. Computers in Industry, 34(3), 271-283. http://dx.doi.org/10.1016/ S0166-3615(97)00046-8.

Tu, Y., Chu, X., \& Yang, W. (2000). Computer-aided process planning in virtual one-of-a-kind production. Computers in Industry, 41(1), 99-110. http://dx.doi. org/10.1016/S0166-3615(99)00006-8.

Turkulainen, V., \& Ketokivi, M. (2012). Cross-functional integration and performance: what are the real benefits? International Journal of Operations \& Production Management, 32(4), 447-467. http://dx.doi. org/10.1108/01443571211223095.
Vernadat, F. B. (1996). Enterprise modeling and integration: principles and applications. London: Chapman \& Hall.

Vollmann, T. E., Berry, W. L., \& Whybark, D. C. (1997). Manufacturing planning and control systems (4th ed.). New York: Irwin/McGraw-Hill.

Voss, C., Tsikriktsis, N., \& Frohlich, M. (2002). Case research in operations management. International Journal of Operations \& Production Management, 22(2), 195219. http://dx.doi.org/10.1108/01443570210414329.

Wu, J., Lu, X., Xu, H., \& Nakagoshi, N. (2013). The method research of production enterprise logistics system integration. In Proceedings of the 2nd International Conference on Energy and Environmental Protection.

Yin, R. K. (2005). Estudo de caso: planejamento e métodos (3. ed.). Porto Alegre: Bookman. 